This is a self-archived version of an original article. This version may differ from the original in pagination and typographic details.

Author(s): Slavov, Matias

Title: Universal Gravitation and the (Un)Intelligibility of Natural Philosophy

Year: 2020

Version: Accepted version (Final draft)

Copyright: ㄷ 2019 University of Southern California and John Wiley \& Sons Ltd

Rights: In Copyright

Rights url: http://rightsstatements.org/page/lnC/1.0/?language=en

Please cite the original version:

Slavov, M. (2020). Universal Gravitation and the (Un)Intelligibility of Natural Philosophy. Pacific Philosophical Quarterly, 101(1), 129-157. https://doi.org/10.1111/papq.12300 
Pre-edited version. Published in Pacific Philosophical Quarterly

https://doi.org/10.1111/papq.12300

\title{
Universal Gravitation and the (Un)Intelligibility of Natural Philosophy
}

\begin{abstract}
This article centers on Hume's position on the intelligibility of natural philosophy. To that end, the controversy surrounding universal gravitation shall be scrutinized. It is very well-known that Hume sides with the Newtonian experimentalist approach rather than with the Leibnizian demand for intelligibility. However, what is not clear is Hume's overall position on the intelligibility of natural philosophy. It shall be argued that Hume declines Leibniz's principle of intelligibility. However, Hume does not eschew intelligibility altogether; his concept of causation itself stipulates mechanical intelligibility.
\end{abstract}

\section{Introduction}

Newton's argument for the law of universal gravitation (LUG) was not readily accepted by early modern philosophers. LUG implies a myriad of utterly strange features. Accordingly, a central issue in the reception of the argument is whether natural philosophical propositions should be intelligible. Leibniz famously criticized Newtonian attraction because it violated his principle of intelligibility (PInt). In the view of Leibniz, the relation between two objects A and B (for example a relation between cause and effect, the relation between a subject/object and a quality, the explanandum and the explananda) is intelligible if and only if we can explain, based on the natures of $\mathrm{A}$ and $\mathrm{B}$, how $\mathrm{A}$ gives rise to $\mathrm{B}$ and why something different from $\mathrm{B}$ does not arise.

In this article, I will focus on Hume's position on the intelligibility of natural philosophy. I shall do this in light of the debate between Newton and Leibniz on universal gravitation. It is widely known that Hume has more sympathies for the former than for the latter. What is not properly understood, however, is Hume's own position on the intelligibility of natural philosophy. To provide an argument on this point, the rest of the article is composed as follows. In the next section, I shall briefly sketch Newton's argument for LUG and highlight its philosophical ramifications. I then explicate Leibniz's PInt, his criticism of LUG and Newton's experimentalist response to the 
criticism. In the subsequent section, I shall first analyze Hume's qualified approval of Newton's experimentalism ${ }^{1}$ and his rejection of PInt. After this I will peruse his rules by which to judge causes and effects. I shall argue that these rules assume mechanical intelligibility.

In conclusion, I formulate the main thesis of this article as follows: Hume rejects PInt but his concept of causation itself stipulates mechanical intelligibility. There is nothing in the nature of an object that would provide some reason why certain properties are predicable of it. Universal gravitation pertains to bodies with mass; there is no ultimate explanation for why this is so. At the same time, Hume's concept of causation instantiates a type of mechanical intelligibility. Causes and effects need to be contiguous, successive and constantly conjoined. These rules take their model from the collisions of bodies or the workings of machines. They impart a mechanistic image of causation; an image that is in tension with a dynamic treatment of phenomena. Hume does not subscribe to Leibniz's PInt, but he assumes a weaker notion of intelligibility, according to which a relation between two objects $\mathrm{A}$ and $\mathrm{B}$ is intelligible if the objects are spatially contiguous and temporally successive.

\section{Newton's argument for LUG and its unintelligible implications}

Newton's argument for LUG is long and intricate. In his interpretation of the argument, Ori Belkind (2012) draws on William Harper's (2016) three stages of the argument. ${ }^{2}$ First, Newton deduces a centripetal concept of force and acceleration from Kepler's area law (Propositions 1-3 in Book 1, Section 2 of the Principia). Second, he deduces the inverse-square proportionality of the centripetal force from Kepler's harmonic rule (Proposition 4). Third, by referring to a plethora of empirical evidence drawn from pendulum experiments and astronomical observations, he affirms that the centripetal force is the force of gravity (Scholium to Proposition 5 in Book 3).

\footnotetext{
${ }^{1}$ The Hume-Newton relationship has by now been thoroughly examined (for example, by Capaldi 1975; Laudan 1981; Jones 1984; Force 1987; Barfoot 1990; Stanistreet 2002; Buckle 2004; Millican 2007; De Pierris 2006; Ducheyne 2009; Randall and Brown 2012; Demeter 2014; Boehm 2016; [author's name omitted]; Hazony and Schliesser 2016). Despite the immense amount of scholarship, Hume's Newtonianinspired criticism of Leibniz on reason and intelligibility has not been given the attention it deserves. ${ }^{2}$ Harper's (2016) paper is in the second edition of the Cambridge Companion to Newton, which was originally published in 2002; that is why the time order seems strange. Smith's (2016) work is also drastically important in this context.
} 
Then Newton connects his third law of motion with gravitation as a universal force of interaction. Consequently, he makes the striking claim that $F_{G} \propto \frac{m_{1} m_{2}}{r^{2}}$, in which $F_{G}$ is the force of gravity, $m_{1}, m_{2}$ are the masses of any two separate bodies, and $r$ is the distance between their centers of mass (Propositions 6 and 7).

The philosophical ramifications of Newton's scientific argument are abundant. Unification of terrestrial and celestial phenomena is arguably the most significant of them. For the present article, I wish to stress one remarkable consequence. To excerpt from Peter Millican (2007), LUG is unintelligible. Whether something counts as intelligible or not is a context-sensitive matter. In Henk W. de Regt's $(2017,40)$ wide-ranging study of scientific understanding, he notes that "theories are not intrinsically intelligible or unintelligible, but intelligible or unintelligible to a particular scientist or group of scientists. In other words, intelligibility is a context-dependent value." The relevant context of this article is the Cartesian mechanistic tradition. It was still the scientific orthodoxy in the early modern period. It was thought, to quote from Belkind $(2012,142)$, that "all physical forces are reducible to mechanical forces of push-and-shove." In the formulation of Katherine Dunlop $(2012,86)$, the Principia did not advance explanations which take as their "model the working of machines," showing how phenomena "result from something like pushing or pulling, localized to the surface of a body." As I will argue in section 1.1, Leibniz did not accept many aspects of Newton's dynamics because it does not conform to such mechanical ideals. Before analyzing Leibniz's views in detail, it is noteworthy to list the unintelligible features related to LUG. From the viewpoint of mechanical philosophy, the astonishing aspects of LUG (in no particular order) can be listed as follows:

Bodies change each other's state of motion through empty space, although there is nothing in between the bodies to count for their changes of motion.

Newton's impressed (that is, causally efficacious) forces include percussion, pressure, and the centripetal force (Principia, Book 1, Definition 4). The first two are contact forces, and perfectly intelligible in the mechanistic paradigm. But Newton adds that one kind of centripetal force is

\footnotetext{
${ }^{3}$ This formula does not appear in any of Principia's editions. Newton did not apply such algebraic equations but proceeded with geometric proofs. Still and all, the proportionality of masses and distance as depicted in the formula is the result of Newton's argument for LUG.
} 
gravity (Definition 5). Therefore, LUG countenances action at a distance, and violates the mechanistic framework.

Gravity penetrates supposedly impenetrable matter (that is packed with corpuscles), because (from a geometric viewpoint) it acts between the center of masses of bodies, not their common boundaries.

Right after Newton admits that he has not explained gravity, and just before he proclaims not to feign hypotheses in the General Scholium, he indicates that there is something strange in gravity's operations that would require more investigation: "Indeed, this force arises from some cause that penetrates as far as the centers of the sun and planets without any diminution of its power to act, and that acts not in proportion to the quantity of the surfaces of the particles on which it acts (as mechanical causes are wont to do) but in proportion to the quantity of solid matter, and whose action is extended everywhere to immense distances, always decreasing as the squares of the distance."

There is no identifiable agent responsible for gravitational motion. Change of motion is initiated without a known agent (like a human being, animal, machine etc.).

To explain the oddity of this point, we may use an example from common experience. Say that a shut door suddenly opens. We naturally assume that such change of motion has some identifiable agent: a human being or an animal, the wind, or a lock mechanism. If we are unable to identify any agent, the workings of gravity resemble paranormal activity: something starts moving or stops moving, yet the agent of the motion is left unspecified. In his famous letter to Bentley, Newton (1692-1693/2004, 102-103) insisted that there is an agent responsible for gravity (McMullin 1978, 297), and he clearly recognizes that a failure to provide a reference to the agent makes LUG utterly weird. Moreover, bodies themselves cannot be the agents, because gravity is an impressed force, and Newton equates impressed forces with actions exerted on bodies: "This force consists solely in the action and does not remain in a body after the action has ceased" (Principia, first Book, Definition 4). The force of gravity is not a property of bodies like inertial mass. 
There is no evidence of a mechanism, like an etherial fluid that is composed of microscopic particles that operate by means of varying density of the fluid, to explain gravity.

Newton provisionally speculates with the idea of a corpuscularian ether in Query 21 of the Opticks. This substance would render LUG intelligible. Due to lack of evidence, he maintains that he does not know what such ether is. Hylarie Kochiras argues $(2011,174)$ that hypotheses like this are legitimate as they are "amenable to empirical investigation" and may furnish experiments, but not because their appeal to make "the world intelligible."

If matter is inanimate and passive, it should not strive towards meaningful goals. How can one piece of matter "know" (or have some intentional, mental propensity) where the other piece of matter is?

Assuming Cartesian metaphysical position on bodies as inert extension, their intentionality seems out of the question. Newton certainly criticizes Descartes' assimilation of space and extension (as his overall substance dualism, as shown by Janiak 2013, section 3 and 4) in his De Gravitatione. But as gravity is not essential to bodies (Principia, Rule 3 in the Rules for the Study of Natural Philosophy), ${ }^{5}$ the salient properties of bodies are the quantities of matter and motion, as well as inertia (Principia, first Book, Definitions 1,2, and 3). None of these resemble mental states. This is echoed in Newton's $(1692-1693 / 2004,102)$ words in his letter to Bentley: "It is inconceivable that inanimate brute matter should, without the mediation of something else, which is not material, operate upon and affect other matter without mutual contact."”

Forces appear only between at least two mass particles. Individual particles do not form a gravity field (that extends to infinity) around them that functions as the agent for the change of motion of another particle.

\footnotetext{
${ }^{4}$ See also Kochiras (2013) for the status of mechanism in Newton.

${ }^{5}$ This relates to the earlier point about forces not remaining in bodies, as they are external actions which cease after the actions themselves cease. Here Newton is clearly opposed to Leibniz's (and later du Châtelet's) notion of living force.

${ }^{6}$ Whether matter is passive or active in Newton is subject to scholarly debate. Recently, Biener has advanced the argument that the sensible qualities of matter are passive, but that there are underlying active powers (Biener 2017, fn. 36 and fn. 54). In his view, this distinction enabled Newton "to separate sensible qualities - understood as the configurations of matter and motion correlated with certain perceptions-from the power, largely unexercised, that enables those configurations to give rise to occurrent experience."
} 
Newton's third law holds that forces are fundamentally interactive. Against Stein's (1970) influential account, Eric Schliesser $(2011,80)$ argues that gravity is a relational (in the Aristotelian parlance accidental, not substantial) "quality of matter that arises through what Newton calls 'the shared action' of two bodies."7 No single body can generate a gravity field. Kochiras' (2017b) position backs up this point: "The gravitational force expressed by his law is a force that is fundamentally relational rather than monadic."

It should be clarified that the aspects listed above are problematic interpretations of LUG. They are not necessarily consequences of Newton's law, Newton's commitments, or even uncontroversial assumptions. Nevertheless, the points are useful for articulating Leibniz's objections to LUG.

\subsection{Leibniz's PInt and his criticism of LUG}

Leibniz did not object to Newton's argument's deductive and empirical structure. The mathematical formulation of LUG is not the problem, either. It is its lack of reason. In his second letter to Clarke, Leibniz $(1715-1716 / 1989,321)$ writes that

...in order to proceed from mathematics to natural philosophy, another principle is required [...] I mean the principle of a sufficient reason, namely, that nothing happens without a reason why it should be so rather than otherwise.

An otherwise sound argument, even as expressed in mathematical terms, is not in itself sufficient for natural philosophy. Natural philosophical principles like LUG require a reason. More precisely, Leibniz's reluctance to accept LUG stems from a deeper source than just lack of a reason. He criticized Newton's argument because it violated what Donald Rutherford (1992) calls his principle of intelligibility. PInt is more restrictive than Leibniz's proclaimed principle of sufficient reason (PSR). To quote from Rutherford $(1992,35)$, Leibniz's commitment to PInt means "that nothing happens for which it is impossible to give a natural reason, i.e., a reason drawn from the

\footnotetext{
${ }^{7}$ For a discussion on Schliesser's position, see Harper (2011, 137-139).
} 
natures of the beings that belong to this world." PInt is echoed by Leibniz's $(1704 / 1996,66)$ own words:

Whenever we find some quality in a subject, we ought to believe that if we understood the nature of both the subject and the quality, we would conceive how the quality could arise from it.

This quote is from his New Essays. He responds to Locke's view according to which it is in God's power to attribute qualities to matter that are not necessarily in its essence. Contrary to Locke's superaddition thesis, Leibniz claims that God does not randomly attach properties to substances. There must be some reason why a given property is predicable of a subject in the natural world (miracles excluded). Such predication is conceivable because of the nature of the being in question. It is the task of PInt to explain how this exactly happens. Rutherford $(1992,36)$ gives a precise formulation of PInt: For any entity and a property that is predicable of the entity, there is a reason why the entity has the specific property, and this reason explains the entity's property in terms of the property "being an 'explicable modification' of the nature of" the entity. Leibniz presumes that it should always be possible to understand how a given quality arises from the nature of a given subject. In this view, nature is intrinsically and ultimately intelligible.

When one applies PInt to LUG, we may see that the latter does not satisfy the former. To show this, we can consider bodies subject to dynamic laws as entities. According to PInt, there should be a reason in bodies' nature of why gravity's properties like long-range action, instant action, penetrability, and seemingly intentional behavior could be attributed to the bodies themselves. Newton did not make any predication like this. He does not explain why the properties follow from the nature of the bodies, or more precisely, how they are its "explicable modifications." 8

Leibniz thinks that Newton's dynamics employs an ontology of powers similar to the Aristotelianscholastic tradition. This is by ascribing attraction - putatively powers in the Aristotelian senseto bodies. Such 'power ascription,' in Leibniz's mind, does not explain anything. To say that bodies attract because of their masses is like saying that fire burns because of its heat. This is as

\footnotetext{
${ }^{8}$ See fn. 4 and 6 of Rutherford (1992, 46-47).
} 
explanatory empty as saying, to paraphrase Molière's famous joke, that opium is soporific in virtue of its dormitive power (Ott 2009, 11). In his article "Against Barbaric Physics," Leibniz (17101716/1989, 314) bashes Newtonian attraction, claiming that it slips "back into barbarism in physics and into the occult qualities of the Scholastics."

Instead of Newton's dynamic physics, Leibniz exalts a paradigmatically mechanistic physics. In the beginning of his "Against Barbaric Physics," he encapsulates what he thinks is the intelligible physics:

That which explains everything in the nature of body through number, measure, weight, or size, shape and motion, which teaches that nothing is moved naturally except through contact and motion, and so teaches that, in physics, everything happens mechanically, that is, intelligibly, this physics seems excessively clear and easy.

For Leibniz, Newton's treatment of the universal force of gravity as an interaction "cannot, at any rate, reduce this attraction to impulse or to intelligible reasons". But "force" à la Leibniz "is exercised only through an impressed impetus" (Leibniz 1710-1716/1989, 317, and 319). Mechanistic physics like this is in accordance with PInt. If we consider "everything in the nature of body," we find quantifiable properties like size, shape, and motion. These properties are clearly attributable to the bodies themselves. In virtue of these attributes we get the measure of how bodies change their state of motion through contact. In mechanistic physics, we do not have the strange, occult quality of attraction that fails to be predicable of bodies - which would ultimately violate PInt.

We might certainly consider attraction to be a force, not a property of bodies. After all, Leibniz's metaphysics is ripe with forces. But he is quite clear that forces, such as the living force, are in bodies. ${ }^{9}$ Attraction should be explained by a reference to the nature of bodies, not by a reference to external forces. Thus he $(1715-1716 / 1989,327)$ writes in his third letter to Clarke:

\footnotetext{
${ }^{9}$ For an analysis of the relationship between living and dead forces within active forces, see McDonough (2014, Section 3.2). His reading seems to support the point that Leibniz thinks forces are in bodies / properties of bodies, and this renders them intelligible and consonant with the mechanical philosophy:
} 
If God wanted to cause a body to move free in the aether round about a certain fixed center, without any other creature acting upon it, I say it could not be done without a miracle, since it cannot be explained by the nature of bodies. For a free body naturally recedes from a curve in the tangent. And therefore, I maintain that the attraction of bodies, properly so called, is a miraculous thing, since it cannot be explained by the nature of bodies.

In the quote above, Leibniz repeats the demand of explaining attraction "by the nature of bodies." This is unlike Newton, who denies that gravity is essential to matter. It should be emphasized that Leibniz does not criticize the first and the second parts of Newton's argument for LUG. ${ }^{10}$ In his view, the crucial mistake is the argument's conclusion. In the final step of the argument in Proposition 7 of the third Book, Newton makes the sweeping claim that the force of gravity "is proportional to the quantity of matter in each [body]." He adds in Corollary 1 of the Proposition that as the bulk of the matter attracts, all mass points (instead of the surfaces of bodies) attract: "For the force of the whole will have to arise from the forces of the component parts." Accordingly, Newton thinks that the third law is a universal law of interaction; any particle attracts every particle with equal and opposite force. ${ }^{11}$

Leibniz thought that by treating forces as inherent powers of bodies tied inextricably to their ability to move and be moved, his own conception of force could preserve the intelligibility that was the great hallmark of mechanism, while nonetheless improving upon the work of those such as Huygens, Descartes and Galileo. For, by Leibniz's lights, the active and passive forces he postulates not only render physics itself more accurate and consonant with reason but at the same time set the stage for its intelligible grounding in his own deeper metaphysics. (Ibid.)

${ }^{10} \mathrm{He}$ accepts the inverse-square magnitude of the centripetal force. He partly praises Newton's argument by referring to it as a "beautiful discovery." Moreover, shortly after the publication of the original edition of the Principia in 1689, Leibniz went on to write an article "Attempt to Explain the causes of Celestial Motions" (Tentamen de Motuum Celestium Causis). Although in this work he rejects the universal character of Newtonian attraction, he nevertheless tries to reconcile the mathematical proof of the inverse-square force and Kepler's laws with his own version of the vortex theory of planetary motions (Kochiras 2017b). For the interplay between Leibniz's and Newton's astrophysics, see Meli (1993, chapter 9).

${ }^{11}$ In Proposition 7 of Book 3 of the Principia, Newton claims that

Further, since all the parts of any planet A are heavy [or gravitate] toward any planet B, and since the gravity of each part is to the gravity of the whole as the matter of that part to the matter of the whole, and since to every action (by the third law of motion) there is an equal reaction, it follows that planet B will gravitate in turn toward all the parts of planet A, and its gravity toward any one part will be to its gravity toward the whole of the planet as the matter of that part to the matter of the whole. [Italics added by the author] 
Leibniz maintains that such universalization lacks justification. By referring to Newtonians, he $(1710-1716 / 1989,314)$ points out that

they even fabricate what they cannot prove through phenomena, for so far, except for the force by which sensible bodies move toward the center of the earth, they have not been able to bring forward any trace of the general attraction of matter in our region.

Here we can see a qualified approval of Newtonian physics: "bodies move toward the center of the earth" because of the centripetal force. But Leibniz considers "mutual gravitation of the planets" to be a fabrication of evidence for the supposed explanation of astronomical phenomena. He $(1710-1716 / 1989,314)$ notes that "we must be careful not to proceed from a few instances to everything." His skepticism of Newton's generalization rests on comparable cases. Unwarrantedly, Gilbert argued that magnetic attraction occurs everywhere, and the alchemist attributed suspicious chemical composition to everything. Leibniz treats Newton's universalization of gravity in the same way. Newton was unable to demonstrate that universal attraction exists between every particle in the universe.

\subsection{Newton's experimentalist response}

In the General Scholium, which was added to the second and third editions of the Principia, Newton responds to the criticism he faced. ${ }^{12} \mathrm{He}$ begins the General Scholium by repudiating two core creeds of the mechanical philosophy: vortex hypothesis of planetary motions, and plenism (earlier, at the end of Book 2 he discusses the mathematics of vortex theories, and cometary orbits at the end of Book 3; the General Scholium highlights these difficulties). The vortex hypothesis is inconsistent with Kepler's laws that ascribe approximately elliptical motion to satellites. The hypothesis also fails to explain the eccentric and regular orbits of comets around the Sun; the

\footnotetext{
${ }^{12}$ Shapiro (2004) notes that Newton restrained from using the term "experimental philosophy" until the publication of the second edition of the Principia in 1712. Before that he highlighted the mathematical structure of his work. The experimental method and reasoning are introduced precisely in the context in which he rebuts the views of his mechanist predecessors (like Descartes) and interlocutors (like Leibniz). Also, as Schliesser notes (2013, section 3.1), Newton made at least nine non-trivial changes between the editions to avoid the charges of Epicureanism.
} 
comets simply do not wander around in different solar systems. ${ }^{13}$ Space between the planets is not a plenum but empty and devoid of air. This can be extrapolated from Boyle's air pump experiment, pendulum experiments with dissimilar materials, and from the laws of motion. There is no evidence of any circling bands holding planets in their orbits, plain and simple.

Although Newton disputes the central tenets of the rival mechanistic physics, he acknowledges that his dynamics is incomplete. He did not say the last word about gravity. In his often-quoted words of the General Scholium: "I have not as yet been able to deduce from phenomena the reason for these properties of gravity, and I do not feign hypotheses." For Leibniz, this won't do. Before establishing that a law expressed in mathematical terms conveys information about nature, ${ }^{14}$ that law needs to be intelligible. There should be a reason in bodies' nature that explains why gravity's various bizarre properties are predicable of them. But Newton admits that he is not able to provide a reason like this. His argument for LUG simply does not meet the standards of Leibniz's PInt.

${ }^{13}$ By comets "wandering" around different stars I am referring to Descartes' figure Plate 6 in his Principles of Philosophy.

${ }^{14}$ Newton separates forces considered from a mathematical point of view, and forces of nature (Stein 1990). $\mathrm{He}$ is very careful on how to translate a mathematical treatment into a physical one. In the explanatory paragraph to Proposition 57 of Section 11 of the first book of the Principia, he notes that

Up to this point, I have been setting forth the motions of bodies attracted toward an immovable center, such as, however, hardly exists in the natural world [...] I now go on to set forth the motion of bodies that attract one another, considering centripetal forces as attractions, although perhaps - if we speak in the language of physics - they might more truly be called impulses. For here we are concerned with mathematics; and therefore, putting aside any debates concerning physics, we are using familiar language so as to be more easily understood by mathematical readers.

At first sight, it is striking how cautious Newton seems to be in translating a mathematical argument into a physical one before his statement in the Scholium to Section 11 of Book 1. He says that centers of mass do not exist in nature - there are no physical correspondents for such infinitesimal ideal points. What is more, in Definition 8 of the first Book, he declines attributing "any sort of propensity toward a center," as Newton considers

these forces not from a physical but only from a mathematical point of view. Therefore, let the reader beware of thinking that by words of this kind I am anywhere defining a species or mode of action or a physical cause or reason, or that I am attributing forces in a true and physical sense to centers (which are mathematical points) if I happen to say that centers attract or that centers have forces.

Such prudence is perhaps due to the infamous connotation of the term 'attraction.' To Newton's readers 'attraction' sounds something like 'propensity.' This might have been equated with a rollback to Aristotelian philosophy in which beings, including bodies, have purposeful goals. In geometric parlance, we may say that the centers of mass attract. In physics this simply means that the bulk of matter, every single particle of a body, attracts another bulk, its every single particle. 
Before devising the General Scholium, Newton was certainly puzzled by the oddity of nonmediated instant causal action. On the one hand, he treats the force of gravity as a real cause of change of motion. ${ }^{15}$ On the other hand, he finds causal action across empty space troubling. Forces are the causes of accelerations, but the way gravity force produces acceleration without contact seems to result into "so great an absurdity that I believe no man who has in philosophical matters a competent faculty of thinking can ever fall into it," as he conceded in his correspondence with Bentley in early 1690's (Newton 1692-1693/2004, 102-103; [author's name omitted]). ${ }^{16}$

Newton recognizes that the implications of LUG are mysterious, and that his argument has been vehemently criticized because of its peculiarity. What is his response to the unintelligibility accusation? In my rendition, an important characteristic in his response-and in his famous line of Hypotheses non fingo, respectively - is to decline an intelligibility requirement like the one expressed in Leibniz's PInt. ${ }^{17}$ Newton refuses to contrive hypotheses just to make the world intelligible. He thinks "that we are," [author's name omitted] interprets, "in no position to stipulate that nature is intelligible." After all, who says that nature should be intelligible? Why should it conform to our pre-reflexive, pre-scientific intuitions of how bodies move? Why should such intuitions be the model for a universal law of motion?

Newton bases his answer to the intelligibility critique on his experimentalism. In a 1713 letter to Cotes, he spells out his experimentalist position against the hypothetical and speculative branch of natural philosophy. Newton $(1713 / 2004,120-121)$ claims that

\footnotetext{
${ }^{15}$ This is apparent already in "De Gravitatione's" Definition 5, in which Newton writes: "Force is the causal principle of motion and rest." In the Principia (Axioms, or Laws of Motion), Newton's original formulation of his second law of motion applies the concept of 'impressed force.' He is also very clear on the causal efficaciousness of forces in the Scholium to the Definitions of the first Book. According to Janiak (2007, fn. 1), "Newton's own conception of force" is this: "a given force F is real iff F stands in some causal relation with some body."

${ }^{16}$ As Henry (2011) argues, the letter to Bentley does not rule out action at a distance. "Inconceivability" and "absurdity" in this context do not mean impossibility. My argument suggests that Newton thought that distant instant action is unintelligible, but this is not a valid argument against LUG, because intelligibility is not a necessary requirement for knowledge about the physical world.

${ }^{17}$ This does not exclude the possibility of Newton changing the standards of intelligibility (see Kochiras' 2013) position. Also, my interpretation does not rule out any reason for gravity's actions (the ether hypothesis in the Opticks, if successful, would render LUG intelligible). The point is that Newton does not accept Leibniz's reasons for rejecting the conclusion of the argument for LUG that relies on PInt.
} 
Experimental philosophy reduces phenomena to general rules and looks up on the rules to be general when they hold generally in phenomena [...] Hypothetical philosophy consists in imaginary explications of things and imaginary arguments for or against such explications, or against the arguments of experimental philosophers founded upon induction. The first sort of philosophy is followed by me, the latter too much by Descartes, Leibniz, and some others.

Here we may zero in on Newton's reference to "imaginary arguments." Descartes' vortex hypothesis of planetary motions, for example, "consists in imaginary explications of things and imaginary arguments for" it. The vortex hypothesis is framed only to make the world intelligible. Speculation with vortexes is not based on anything empirical (or mathematical ${ }^{18}$ ). No astronomical observations indicate its existence. We are not able to carry out any experiment in which some postulated properties of the Cartesian vortexes could be used as salient variables (e.g., we cannot collect pieces of vortexes in a jar, heat it up and observe the consequences). ${ }^{19}$ "For hypotheses ought to be applied," Newton (1672/1974, 5-6) stated already in his early pre-Principia correspondence with Oldenburg, "only in the explanation of the properties of things, and not made use of in determining them; except in so far as they may furnish experiments." This suggests that for Newton, provisional hypotheses belong to natural philosophy but not hypotheses that forcefully try to make the nature intelligible for us. However, in his correspondence with Oldenburg in 1675, he articulates a more mitigated view that allows a limited role of intelligibility:

And therefore considering that such an Hypothesis would much illustrate ye papers I promis'd to send you, \& having a little time this last week to spare: I have not scrupled to describe one so far as I could on a sudden recollect my thoughts about it, not concerning

\footnotetext{
${ }^{18}$ It is interesting how qualitative Descartes' vortex hypothesis in his Principles is compared to Newton's mathematical astrophysics. The vortex hypothesis does not encompass any interconnected mathematical proportions (unlike Descartes' impact rules). As Chris Smeenk (forthcoming) explains, Descartes did not imagine the planetary orbits to be stable, so he had no need for a predictive, quantitative astrophysics like Newton.

${ }^{19}$ Experimenting with vortexes might not be entirely impossible. Descartes' vortex theory is only speculative, but Huygens devised an experiment in 1667 with cylindrical vessel filled with water (representing the etherial subtle substance) and pieces of sealing wax (that represent astronomical bodies). This was an experiment that he used to explain gravity by positing spherical vortexes (Snelders 1989, 212213).
} 
my self whether it shall be thought probable or improbable so it do but render ye papers I send you, and others sent formerly, more intelligible (Quoted in Westfall 1993: 103).

Although Newton's take on hypothesis might have somewhat changed during his life, it can be said that intelligibility is not an explanatory constraint for him as it is for Leibniz. He does not propound to explain gravity. The universal force explains a good deal of its effects, including free fall, trajectories, the tides, satellite, planetary and cometary orbits. But he does not know the cause of this force: "Thus far I have explained the phenomena of the heavens and of our sea by the force

of gravity, but I have not yet assigned a cause to gravity" (General Scholium, Principia). ${ }^{20}$ Despite not explaining the nature of universal gravity, there is still a considerable amount of convergent empirical evidence from pendulum experimentation and astronomical observations in favor of the argument for LUG. This evidence is against Leibniz's alternative mechanistic physics that conforms to PInt.

\section{Hume on intelligibility}

In this section, I proceed to analyze Hume's position on the intelligibility of natural philosophy. I submit that it is twofold. I shall do this by first expounding on how Hume, roughly and broadly, sides with Newton's experimentalism and eschews Leibniz's PInt. Then I will show that Hume still retains a residue of mechanical intelligibility. My argument propounds that Hume denies the strong intelligibility constraint as expressed by Leibniz's principle. Still he adopts a weaker but positive notion of intelligibility. His weak commitment is consistent with Leibniz's ideal of mechanistic physics.

\footnotetext{
${ }^{20} \mathrm{He}$ did, nevertheless, devise provisional hypotheses. These hypotheses include a reference to ether and corpuscles. Newton publicly presented his corpuscular hypothesis to the Royal Society in mid-1670's and his ether hypothesis in the Queries of the Opticks later at the beginning of the $18^{\text {th }}$ century. But in his official stance he did not "need to," as Iliffe and Smith $(2016,2)$ put it, "have recourse to as yet unconfirmed underlying entities."
} 


\subsection{Hume's experimentalism and his rejection of PInt}

Hume's experimentalism is most clearly present in the Introduction to his Treatise (in paragraphs 8-10; SBN xvii-xviii). His premise is this: whether we are dealing with bodily or mental phenomena, their essences are unknown to us. To acquire any notions of the properties of bodies, we must carry out "careful and exact experiments." And although we should, Hume continues,

endeavour to render all our principles as universal as possible, by tracing up our experiments to the utmost, and explaining all effects from the simplest and fewest causes, 'tis still certain we cannot go beyond experience.

Hume is clear that "we can give no reason for our most general and most refined principles, beside our experience of their reality." Hypotheses that strive for ultimate explanations-putative explanations that address why something is the way it is, rather than otherwise-_ought at first to be rejected as presumptuous and chimerical." Hume freely confesses "his ignorance" of ultimate explanations and wishes to avoid "that error, into which so many [hypothetical and speculative philosophers like Descartes and Leibniz] have fallen, of imposing their conjectures and hypotheses on the world for the most certain principles." "None" of the special sciences "can go beyond experience, or establish any principles which are not founded on that authority."

Given Hume's announced experimentalism, one may ask: is Hume leaning on Newton? In the Introduction, he does not explicitly refer to him, or to any of his works. Both Steffen Ducheyne (2009) and Eric Schliesser (2009) have evaluated Hume's supposed Newtonianism critically. ${ }^{21}$ They note that terms 'experiment' and 'experimental' were used widely in many seventeenth- and eighteenth-century textbooks of natural philosophy. Because references to these 'experiments' were abundant in the scientific culture of Hume's time, there is no evidence that Hume was essentially a Newtonian philosopher.

\footnotetext{
${ }^{21}$ Concerning Schliesser's position on Hume on experiment, Taylor's (2015, Chapter 1) contribution is a useful corrective to it.
} 
We may also catch a glimpse of Ephraim Chambers' Encyclopedia from the year 1728. Newton is mentioned under the rubric 'Experimental' in Chambers' $(1728,368)$ dictionary definition, but also Bacon and Boyle are listed under the title. Moreover, the dictionary definition includes institutions like the Royal Society, the Royal Academy of Paris and Accademia del Cimento. There is nothing especially Newtonian about articulating an experimental philosophy.

Furthermore, what Hume means by the word 'experiment' is closer to the meaning of ordinary experience rather scientific experimentation (Schliesser 2007, Section 4.1). Typically, 'experiment' (such as Newton's pendulum experimentation with disparate materials, as he reports it in Proposition 6 of Book 3 of the Principia) denotes a highly idealized target-system. This restricted system incorporates salient variables that can be manipulated, as well as some variables that need to be neglected (like air resistance). But 'experience,' for Hume, means observation and memory of constant conjunctions of species of objects or events (T 1.3.6.2; SBN 87). It is telling that while experiments always include error estimates - say, the acceleration near the surface of the Earth is $9.80 \mathrm{~m} / \mathrm{s}^{2} \pm x$, in which $x$ denotes an arbitrarily small error estimate-Hume never points out that experimental results apply within a certain margin of error. To the contrary, Hume assimilates experiments in natural philosophy with exactness (T Intro 8; SBN xvii).

The preceding critical observations show that it is not reasonable to assume that Hume's experimentalism is exclusively Newtonian. However, there are important cultural reasons, related to Newton's reputation and reception, that make Hume's Newtonianism plausible. Boyle and Hooke, among others, adopted experimental philosophy in Britain in 1660's. The early members of the Royal Society followed Bacon's methodology. Newton emerged as an authorial figure after the publication of the Principia and during his presidency of the Royal Society. When Hume composed his Treatise and the Enquiries in mid-eighteenth century, many natural philosophers assimilated experimental philosophy with Newton, not so much with Bacon (Anstey 2015).

There are two senses in which Hume's position is akin to Newton's. First, Hume contrasts hypotheses with experiments. Like Hume in Treatise's Introduction, Newton also establishes his experimentalist position to counter hypotheses. In other parts of his work, Hume recognizes that it was Newton in particular who criticized hypotheses to back up his experimentalist natural 
philosophy in opposition to speculative philosophy. In footnote 16 of the first Enquiry (7.25), Hume writes that Newton did not accept the existence of ether on speculative grounds. In his History (Volume 6,542), he notes that Newton was "cautious in admitting no principles but such as were founded on experiment," thus excluding merely hypothetical principles in natural philosophy. As I ([author's name omitted]) have argued elsewhere:

Hume does not think that natural philosophical principles require intelligibility; they can be accepted on experimental grounds, 'however new or unusual' they are. In a Newtonian and anti-Leibnizian fashion, Hume claims that 'we can give no reason for our most general and most refined principles, beside our experience of their reality' (T Intro 9; SBN xviii, my emphasis).

Second, and more importantly, Newton's Rule 3 of the Rules for the Study of Natural Philosophy is very congenial to Hume's experimentalism. Newton argues that we can know the properties of bodies "only through experiments." He considers the impenetrability of bodies: "We find those bodies that we handle to be impenetrable, and hence we conclude that impenetrability is a property of all bodies universally." We gather this "not by reason but by our senses." Hence Newton ends up arguing that the way for us to know about the qualities of bodies is by extrapolating from the observed to the unobserved. Such appeal to the extrapolation of empirical evidence comes close to what we in our present terminology call Humean induction. ${ }^{22}$ Newton writes that any property of a body, like extension or hardness, "is known to us only through our senses, and yet there are bodies beyond the range of these senses; but because" the quality in question "is found in all sensible bodies, it is ascribed to all bodies universally."

Hume could not agree more with Newton's Rule 3. We acquire the ideas of qualities from sensory impressions. ${ }^{23}$ Then by repeated experience we attribute the qualities to given objects. We do not

\footnotetext{
${ }^{22}$ I find this to be an important detail about the similarities of Newton and Hume. For the most part, one could say that Newton is applying or arguing for Aristotelian induction, according to which general laws or principles are deduced from observed instances. This is not so in Rule 3, as the rule considers Humean induction. For the differences between the Aristotelian and Humean inductions, see Millican (2003, fn. 25). ${ }^{23}$ This is evident from Hume's copy principle (T 1.1). Here it suffices to briefly formulate the principle as follows: all simple ideas are caused by correspondent simple impressions.
} 
know, based on our reason, why a certain object is endowed with certain properties. "The central idea of the Newtonian inductive method," that Hume accepts according to Graciela De Pierris (2006, 280),

is that exceptionless or nearly exceptionless universal laws are inductively derived from 'manifest qualities' or observed 'phenomena,' and only further observed phenomena can lead us to revise these laws.

Hume clearly accepts Newton's LUG. In the first Enquiry (6.4; SBN 57-59), he classifies the proposition concerning the law as 'proof.' This renders it a high-class, unexceptional factual proposition. Hume is also clear of the causal efficaciousness of gravity in the second Enquiry (6.6; SBN 236):

There are some causes, which are entirely uniform and constant in producing a particular effect; and no instance has ever yet been found of any failure or irregularity in their operation [...] The production of motion by [...] gravity is an universal law, which has hitherto admitted of no exception [...] philosophers infer, with the greatest certainty, that the moon is kept in its orbit by the same force of gravity, that makes bodies fall near the surface of the earth (EHU 6.4; SBN 57 and EMP 6.6; SBN 236).

To bolster his experimentalism, Hume provides a thought experiment in which Adam, an a priori $^{24}$ epistemic agent is a person with perfect human sensory system and reasoning faculty, but no experience of the course of nature whatsoever (EHU 4.6; SBN 27, Abstract 11-14; SBN 650$652) .^{25}$

${ }^{24}$ In this context, I use the definition of a priori provided by Millican (2007, xxxvii):

What makes a truth a priori is that it can be justified without appeal to experience, purely by thinking about the ideas involved. Matters of fact, by contrast, can be known to be true (or to be false) only by consulting experience.

This definition is challenged by Smit (2010), who argues that Hume adheres to the from-grounds notion of the a priori. See Millican's (2017, fn. 87) response.

${ }^{25}$ Wade Robison (2018) shows how Hume uses Adam to tackle Cartesian rationalism about causation. 
Suddenly, Adam is brought into the world. He sees, for the first time, the following objects in front of him: water, flame, gunpowder, and lodestone. Can he, only by relying on his immediate perception of these objects, and on his reason, infer the respective qualities of these objects? Can he know that water could be the cause of drowning, that flame causes heat, gunpowder explodes after ignition, and lodestone attracts metal? Is he able to ascribe these properties to the objects? Hume's abrupt answer is no. Adam's sensory system and reason is analogous to ours, but he does not have experience like us. There is no reason why being able to cause drowning is a property of a liquid, heat of fire, explosion of powder and attraction of magnet. We can do such predications because of our frequent and collective experience. Because Adam lacks experience, he cannot.

Consider Adam now witnessing scenarios that Newtonian dynamics predicts. Hume invokes two cases in the first Enquiry (4.9; SBN 29). I hold a rock in my hand. I release it. Can Adam know, before the release, that the rock will fall downwards? Does he have any reason for this prediction? Why is it more reasonable to say that the rock falls instead of saying that it goes up, moves sideways, or disappears and instantly appears somewhere else? What about the collision of bodies? When Adam sees, to use the famous billiard ball example, that the cue ball approaches the object ball, can he predict the ensuing motion of the object ball before the collision? Will it stop, bounce, continue along a straight line?

Adam has no prior experience, so he must rely solely on his reason. What reason does he have for choosing among indefinite number of logical possibilities, in this case, the directions of motion? Hume claims there is no such reason:

All these suppositions are consistent and conceivable. Why then should we give the preference to one, which is no more consistent or conceivable than the rest? All our reasonings $\grave{a}$ priori will never be able to shew us any foundation for this preference (EHU 4.10; SBN 30; see also DNR 2.13; SBN 145-6).

Because conceivability entails possibility, everything we can imagine possibly exists ( $\mathrm{T}$ 1.2.2.8; SBN 32; EHU 2.4; SBN 18). Michael Della Rocca (2017, 465-466) argues that this is a metaphysical principle for Hume, which states that conceivability is sufficient for possibility. It is 
relevant for Hume's argument on the nature of causation, especially his disclaim of necessary connection. Here I wish to stress the epistemic relevance of the principle. Thinking alone does not inform us of any causal relations. When we are dealing with the laws of dynamics that predict the change of motion of objects, our only recourse is experience: "all the laws of nature, and all the operations of bodies without exception, are known only by experience" (EHU 4.9; SBN 29). Gravity law gives us the vector quantity of the free fall of an object, and the law of conservation of momentum gives us the final state of an isolated system. ${ }^{26}$ With these previously tested laws we can predict the resultant motion of the object in question. But even after "a thousand experiments" there is "no satisfactory reason" for any such recurring motions (EHU 12.25; 162).

Even an otherwise ideal epistemic agent, if he lacks experience, cannot have any knowledge of what causes what. There is nothing intuitive about the relation of cause and effect. Adam can intuit relations of ideas. He would know immediately that blue is not red, that triangle has three sides, and that the photo of his friend resembles his friend (T 1.3.1.2; SBN 70). ${ }^{27}$ These comparisons are intelligible. To borrow from Peter Dear's $(2006,14)$ definition of intelligibility, we may say that relations of ideas are self-evident. To the contrary, causal relations are not self-evident. Their identification requires experience. In the Treatise (1.3.1.1; SBN 69-70) and the first Enquiry (12.29; SBN 164), Hume puts the point eloquently:

'tis evident cause and effect are relations, of which we receive information from experience, and not from any abstract reasoning or reflection. There is no single phaenomenon, even the most simple, which can be accounted for from the qualities of the objects, as they appear to us; or which we cou'd foresee without the help of our memory and experience.

The existence, therefore, of any being can only be proved by arguments from its cause or its effect; and these arguments are founded entirely on experience. If we reason $\grave{a}$ priori, any thing may appear able to produce any thing. The falling of a pebble may, for ought we know,

${ }^{26}$ It should be added that in this example the vector quantity and the final state of the system are probabilistic, not necessary. In Hume's language, and in accordance with his tripartite definition of knowledge (EHU 6, fn. 10; SBN 56), the predictions based on laws of nature count as 'proofs.' The predictions are not mere 'probabilities' but they do not amount to 'demonstrations,' either.

${ }^{27}$ After acquiring the relevant simple ideas from their simple impressions but without experience. 
extinguish the sun; or the wish of a man controul the planets in their orbits. It is only experience, which teaches us the nature and bounds of cause and effect, and enables us to infer the existence of one object from that of another.

The world in which we live could be so strange that dropping a piece of rock would put out the Sun, or the will of a human being might alter the paths of planets. This discloses the problem of Hume's refutation of reported miracles. Putative miracles are bizarre, and Hume rejects them. In essence, he denies miracles because their probability is extremely lower than the probability of laws of nature. The latter are exceptionless regularities, so if a law-breaking miracle takes place, we should conclude that something as improbable as a violation of exceptionless regularity happens (EHU 10.12; SBN 114-115). Nevertheless, Hume's argument relies on the normative authority of uniform experience (see Boehm 2013), on the application of Bayesian conditional probabilities (see Owen 2003), and on social epistemological considerations about the reliability of witnesses (Dicken 2011), not on the argument that laws are somehow more reasonable or intelligible than exceptions to them.

We do not see rocks extinguishing stars or minds controlling planets because our contingent world is not like this. But there would be no difference between such astonishing putative causal relations and those that are familiar to us. In Hume's view, there is no reason why any given property is predicable of any given object. Two objects or events that are constantly conjoined count as a causal relation. The objects and events "seem entirely loose and separate" (EHU 7.26; SBN 74). One thing or event follows another. This is causation for Hume. "The constant conjunction of objects determines their causation," he argues (T 1.3.15.1; SBN 173).

Given Hume's denial of intelligibility of causal relations, we may now see how his position is in opposition to PInt. Ascribing qualities to objects is not founded on reason. We may conceive that objects have an indefinite amount of properties. ${ }^{28}$ There is nothing in the nature of the body that is responsible for the properties that are predicable of it. Accordingly, there is no reason why

\footnotetext{
${ }^{28}$ Hume thinks there is one metaphysical requirement: it is impossible that objects have self-contradictory qualities. We cannot conceive a mountain without a valley, or a round triangle, because these are not only inconceivable but also metaphysically impossible (T 1.2.2.8; SBN 32).
} 
universal gravitation is attributable to bodies as Leibniz's PInt requires. ${ }^{29}$ Hume's Newtonianinspired point is that there does not need to be any reason like this. The way gravity causes changes of motion is extremely weird. Millican (2007, xxix) hits the nail right on the head: Hume undermines "even the ideal of causal intelligibility." Alexander Rosenberg's (1993, 73) reading supplements Millican's position:

the whole notion that causation rests on or reflects the intelligibility or rationality of sequences among events is a mistake. Accordingly, for Hume, the aim of science cannot be to reveal the intelligible character of the universe, but simply to catalogue the regularities that causal sequences reflect.

An utterly strange effect might follow a common cause - "The falling of a pebble may, for ought we know, extinguish the sun; or the wish of a man controul the planets in their orbits" (EHU 12.29; SBN 164)-because causation is a regular, not an intelligible relation.

Additionally, Hume rejects Leibniz's PInt because it subsumes PSR. The latter principle states that there is a reason why something is the way it is, not otherwise. This statement encompasses a need for an ultimate, not just fundamental, explanation. In his article "On the Ultimate Origination of Things," Leibniz $(1693 / 1989,149)$ claims that "however far back we might go into previous states [of the world], we will never find in those states a complete explanation [ratio] for why, indeed, there is any world at all, and why it is the way it is." The ultimate reason for everything in the world is an extramundane God. He created the substance of monads. These monads do not have parts and cannot be divided to infinity. God and monads provide an ultimate theological and metaphysical explanation for all physical phenomena, like causation, motion, space, time and bodies (Burnham 2018, Ingress and Section 8).

Hume disagrees with "explaining ultimate principles" (T Intro; SBN xviii) in this way. For him, inquiry is open-ended. He claims that "the most perfect philosophy of the natural kind only staves

${ }^{29}$ Although this article focuses on Hume, it should be mentioned that in assessing gravity's relation to bodies, Locke's position is clearly similar to Newton's. Both deny that gravity is essential to bodies (Kochiras 2017a, 4.2). 
off our ignorance a little longer" (EHU 4.12; SBN 31). For example, gravitational attraction could be explained by an etherial fluid that is composed of corpuscles. Newton considers a similar kind of provisional hypothesis in Query 21 of his Opticks. ${ }^{30}$ Perhaps, ex hypothesi, depending on the varying density of the fluid, gravitational acceleration is greater in the space which is devoid of corpuscles, like near the surfaces of planets, and lesser in the area between planets, which is dense with corpuscles. Such fluid would provide a mechanism that explains the motions of astronomical objects and explains away the quirky distant action inherent in Newton's dynamics. Hume even praises such hypothesis in the first Enquiry (EHU 7.25; SBN 73, fn. 16). In his view, Newton

had recourse to an etherial active fluid to explain his universal attraction; though he was so cautious and modest as to allow, that it was a mere hypothesis, not to be insisted on, without more experiments.

Although the ether hypothesis would (given that it could be experimentally processed) amount to an explanation, it would not be an ultimate explanation. If we say that corpuscles are the ultimate building blocks of the universe, we can still ask a further question: what is the composition of these corpuscles? Are there sub-corpuscularian particles? Search for an ultimate level of explanation would lead to a never-ending quest. We could still go on and inquire into the composition of the sub-particles, and so on.

If my interpretation of Hume is correct, he shuns putative ultimate explanations because they mark an end to investigation. This line of interpretation might still be challenged in the following way. One could object that Hume also marks an end to investigation. To think about or believe in anything, we need to have ideas. The minimally sensible discrete items are needed for us to have simple ideas. Accordingly, ideas are thoughts that are required for any type of investigation: scientists in any discipline need to think and make inferences. ${ }^{31}$ This reservation concerning the scope of inquiry stems from the copy principle, which clearly states that simple impressions cause simple ideas, and the latter resemble the former in all their intrinsic features (Landy 2018, 20-28).

\footnotetext{
${ }^{30}$ But Newton deliberately denies having any knowledge of this fluid: "for I do not know what this Ather is." (Opticks, Query 21).

${ }^{31}$ For this point, see Boehm's (2016) foundational project reading.
} 
However, when it comes to the microstructure of matter, Hume does not stipulate anything about the minimal size of the putative microscopic particles, or what their sub-composition could be, or how many sub-levels particles might have. Particles that are currently imperceptible for us might become observable with the advancement of microscopic technology. Hume notes that we could "form ideas, which shall be no greater than the smallest atom of the animal spirits of an insect a thousand times less than a mite" (T 1.2.1.5; SBN 28). So what counts as minimally sensible depends on the current technology available. Microscopic and telescopic technologies enlarge our sensory perception (T 1.2.1.4; SBN 27-28):

A microscope or telescope, which renders them [minute parts of bodies] visible, produces not any new rays of light, but only spreads those, which always flow'd from them; and by that means both gives parts to impressions, which to the naked eye appear simple and uncompounded, and advances to a minimum, what was formerly imperceptible.

The copy principle does not mark an end to our investigations in atomic physics. ${ }^{32}$ This includes the ether hypothesis as a potential explanation of gravitational motion. We do not know beforehand whether our inquiries in natural philosophy will eventually come to a halt. Unlike Leibniz, Hume does not believe in an ultimate metaphysical or theological foundation for natural philosophy. Such non-empirical school metaphysics and divinity should be committed to the flames as they "contain nothing but sophistry and illusion" (EHU 12.34: SBN 165). Hume does not subscribe to PSR, and subsequently, to PInt. Here we may again see a connection with Newton's position. In referring to Newton, Hume points out that his laws do not "pretend to assign the ultimate cause of any natural operation" (EHU 4.12; 30). This is consistent with Newton's modesty about not assigning a cause to the force of gravity in the General Scholium ([author's name omitted]). ${ }^{33}$

\footnotetext{
${ }^{32}$ Berkeley anticipates this in his New Theory of Vision (85):

For when we look through a microscope we neither see more visible points, nor are the collateral points more distinct then when we look with the naked eye at objects placed in a due distance. A microscope brings us, as it were, into a new world: it presents us with a new scene of visible objects quite different from what we behold with the naked eye.

For a discussion on this point, see Hight $(2008,157)$.

${ }^{33}$ See Ducheyne's (2012, 22-25) study of Newton's hierarchic structures of causal processes. In the General Scholium, Newton however argues for an ultimate cause, which is a theistic God. This theological argument is immune to the revisions from physics (Janiak 2008, 13). In this respect, Newton and Hume are very
} 


\subsection{Hume's reliance on mechanical intelligibility}

Although Hume rejects Leibniz's PInt, and by and large supports Newton's experimentalism, it would be wrong to claim that he eschews intelligibility altogether. I think Millican (2007, xxix) goes too far in claiming that "even the supposed intelligibility of causation by mechanical impact (e.g. of billiard balls) is an illusion, generated by familiarity." In what follows, I shall challenge one aspect of the preceding view. I argue that for Hume "mechanical impact (e.g. of billiard balls)" is a paradigm example of causation. His concept of causation assumes a mechanism. The assumption of mechanism partly renders causation intelligible. Accordingly, there is a type of intelligibility incorporated in his philosophy of causation. As is evident by the previous section, this is not based on any inflationary metaphysics which suggests that modifications of objects are explainable by the natures of the objects themselves. Hume does not share Leibniz's premise according to which nature is ultimately intelligible. Instead, he subscribes to a weaker premise: mechanical intelligibility of causation.

To properly assess such intelligibility, it is noteworthy to quote paragraph 9 of the Abstract of the Treatise in its entirety. In the lengthy paragraph, Hume examines "as perfect an instance of the relation of cause and effect as any which we know," that is, the paradigm example of causation:

Here is a billiard-ball lying on the table, and another ball moving towards it with rapidity. They strike; and the ball, which was formerly at rest, now acquires a motion. This is as perfect an instance of the relation of cause and effect as any which we know, either by sensation or reflection. Let us therefore examine it. 'Tis evident, that the two balls touched one another before the motion was communicated, and that there was no interval betwixt the shock and the motion. Contiguity in time and place is therefore a requisite circumstance to the operation of all causes. 'Tis evident likewise, that the motion, which was the cause, is prior to the motion, which was the effect. Priority in time is therefore another requisite circumstance in every cause. But this is not all. Let us try any other balls of the same kind in a like situation, and we shall always find, that the impulse of the one produces motion in the

different. Hume certainly does not accept the argument from design as Newton puts it in the General Scholium. 
other. Here therefore is a third circumstance, viz. that of a constant conjunction betwixt the cause and effect. Every object like the cause, produces always some object like the effect. Beyond these three circumstances of contiguity, priority, and constant conjunction, I can discover nothing in this cause. The first ball is in motion; touches the second; immediately the second is in motion: and when I try the experiment with the same or like balls, in the same or like circumstances, I find, that upon the motion and touch of the one ball, motion always follows in the other. In whatever shape I turn this matter, and however I examine it, I can find nothing farther.

Regarding causation, the essentials of the preceding quote can be summarized in three points. Cause and effect need to be 1) contiguous, 2) successive, and 3) constantly conjoined. All requirements are codified in the rules by which to judge causes and effects in the first Book of the Treatise (T 1.3.15; SBN 173-176). The rules provide a reason to exclude regularities that are not causal. ${ }^{34}$ My argument suggests that in these rules we may see Hume's assumption of mechanical intelligibility (this point is anticipated by Schliesser 2007, section 4.4; 2009, 192).

Before making an argument on Hume's reliance on mechanical intelligibility, it is necessary to provide at least some definition of the term 'mechanical philosophy.' Part of the difficulty in defining 'mechanical philosophy' is that it is not an explicit, well-defined term. It has various interconnected meanings. ${ }^{35}$ For the present article, a relevant sense of 'mechanical' is the one with

\footnotetext{
${ }^{34}$ As constant conjunctions lack intelligibility, any object or event might be a cause to another object or event, which is its effect. For example, 'the night is followed by the day' is a true regularity about the world, but the relation is not a causal relation.

${ }^{35}$ Garber and Roux (2013, xi) identify four enterprises that stand for 'mechanical philosophy.' In their definition, 'mechanical philosophy' means

(1) the general program of substituting for the "common philosophy," i.e. the scholastic philosophy, a new philosophy, still to be identified; (2) the more specific rejection of Aristotelian hylemorphism and the correlated adoption of an ontology according to which all natural phenomena can be understood in terms of the matter and motion of the small corpuscles that make up the gross bodies of everyday experience alone; (3) the comparison of natural phenomena, most specifically the world and animals, to existing or imaginary machines; (4) lastly, the ontology associated with mechanics as a new mathematical science of motion, the laws of which are described as the laws of nature in general.

Garber and Roux also point out that although in some contexts the different enterprises were connected, one could be a mechanical natural philosopher by subscribing to one of the four points, not necessarily to all of them.
} 
which Cartesian and Leibnizian physics operate. To quote from Millican (2007, xv), "Cartesian physics" operates "exclusively by means of mechanical causation: interaction between contiguous parts of matter by pressure and impact." For his part, Leibniz argues that in mechanistic natural philosophy "nothing is moved naturally except through contact and motion," so "in physics, everything happens mechanically, that is, intelligibly" (Leibniz 1710-1716/1989, 312). Below I argue that it is this notion of mechanistic intelligibility that Hume tacitly assumes in his philosophy of causation.

\subsection{1 "Contiguity in time and place is therefore a requisite circumstance to the operation of all causes"}

Newton's laws countenance action at a distance. This is at odds with mechanical philosophy, since according to it change of motion is initiated by impulse in the collision of bodies. In the Treatise, Hume thinks that contiguity is indispensable for bodily causation. ${ }^{36} \mathrm{He}$ is highly skeptical of action at a distance (T 1.3.2.6; SBN 75):

Tho' distant objects may sometimes seem productive of each other, they are commonly found upon examination to be link'd by a chain of causes, which are contiguous among themselves, and to the distant objects; and when in any particular instance we cannot discover this connexion, we still presume it to exist. We may therefore consider the relation of contiguity as essential to that of causation.

Here Hume leans on Cartesian cosmology. Consider Descartes' plenism as he formulates it in his Principles. There is no empty space independent of bodies. On that account, there is no action at a distance as there is no vacuum between the causally related bodies. ${ }^{37}$ For Hume, bodies change

\footnotetext{
${ }^{36}$ By referring to bodily causation I deliberately exclude Hume's maxim in the Treatise, which states "that an object may exist, yet be nowhere" ( $\mathrm{T}$ 1.4.5.10; SBN 235). There are objects of perceptions that are not physical objects but may still be causally related.

${ }^{37}$ Or at least we do not have the idea of a vacuum. Hume's Cartesian conclusion is that "'tis impossible to conceive either a vacuum and extension without matter" (T 1.2.4.2; SBN 40) and that "we can form no idea of a vacuum, or space, where there is nothing visible or tangible" (T 1.2.5.1; SBN 53). Similarly, Descartes formulates his philosophy of space in Principles 2.11 and 2.16:

If we concentrate on the idea which we have of some body [...] we shall easily understand that the same extension which constitutes the nature of body also constitutes the nature of space [...] That a
} 
their state of motion at contact by impulse. ${ }^{38}$ His mechanistic position is clearly in tension with a dynamic treatment of force and motion, ${ }^{39}$ which allows forces to operate at a distance. When Hume presents his paradigm case of causation, the collision of bodies, he summarizes his view as follows: "Motion in one body is regarded upon impulse as the cause of motion in other" (T 1.3.2.9; SBN 76-77).

Nevertheless, Hume's contiguity rule could mean something more inclusive than just contact. It could be interpreted to stand for next-to relation. I am contiguous with my neighbor, although we are not standing shoulder to shoulder all the time (or at any time). For two objects to be contiguous, it suffices that the one is next to the other. In addition, cause and effect might relate to events rather than to objects. Although two bodies need to touch each other to be causally related, events are contiguous in the sense that a causing event is next to the effected event. Could this make Hume's concept of causation consistent with Newton's dynamics? For example, the centripetal force of gravity, the causing event, is contiguous with the acceleration it produces, the effected event. The Moon, or a rock released from my hand do not need to touch the Earth. Still the force of gravity causes both to fall toward the Earth.

But even this model of causation is inconsistent with Newton's dynamics, as Hume's argument for succession rule indicates.

vacuum in the philosophical sense of the term (that is, a space in which there is absolutely no [material] substance) cannot exist is evident from the fact that the extension of space, or of internal place, does not differ from the extension of body.

There are of course notable differences between Hume and Descartes: For Descartes, space is extension which is an attribute of the substance of body, whereas Hume thinks that we acquire the idea of extension from visual and tactile impressions. Still and all, both end up assimilating space with extension.

38 Malebranche's Search after Truth (1674-1675/1977) probably influenced Hume in this respect. According to Lisa Downing (2005), Malebranche was committed to a rigorous mechanical philosophy in which bodies interact only by means of contact.

${ }^{39}$ Moreover, Hume's and Descartes' positions are also similar in a sense that for their natural philosophies 'force' is not a key concept. Both emphasize the causal efficaciousness of motion and impact. This is different from Newton, as for him net force is responsible for all accelerations. 


\subsection{2 "Priority in time is therefore another requisite circumstance in every cause"}

The Enquiries drop the contiguity rule. They nevertheless retain the succession rule. The rule states that the cause comes before the effect. Hume seems to think along the following lines. When I release a rock, the releasing is an event that precedes the event of falling. This is inconsistent with Newton's dynamics, in which force and acceleration are simultaneous. Force, the cause, is not an object or an event that temporally precedes acceleration, the effect. The three laws of motion and the law of universal gravitation do not encompass succession. The centripetal force of gravity and the resultant acceleration do not form a cause and effect pair in which the former takes place before the latter. Instead, universal gravitation is constant and instant.

Hume does not claim that contemporaneous causation is impossible. Rather, he provides a reduction to absurdity argument (Ryan 2003). If we allow that "one cause were co-temporary with its effect, and this effect with its effect, and so on, 'tis plain there wou'd be no such thing as succession, and all objects must be co-existent" (T 1.3.2.7; SBN 76). The problem with simultaneity in causation is the destruction of succession, and hence time. This would be an absurdity. Thus there is a type of intelligibility embedded in Hume's concept of causation-cause and effect must be linearly ordered in time, otherwise causation would not make sense. Although he does not utilize Leibniz's PInt, his case for temporal priority of causes rests on a type of intelligibility requirement. Both Leibniz and Hume argue for some constraints on natural philosophy. The reduction to absurdity argument discloses a rationalist side of Hume the archempiricist. As Leibniz thinks gravity without PInt does not make sense, Hume thinks causation without a mechanism does not make sense. The conclusion of Hume's argument on priority of causes over effects is more apt to Leibnizian mechanistic physics than to Newtonian dynamics.

\subsection{3 "Here therefore is a third circumstance, viz. that of a constant conjunction betwixt the cause and effect"}

For Hume, causation is constant conjunction among species of objects or events. One thing or event is followed by another. This is inconsistent with a causal formulation of Newtonian 
dynamics. Newton models nature in terms of interactions among mass particles. His dynamic view is considerably different compared to a philosophy that displays the world as an intricate machine. But this is one of the core assumptions of mechanical philosophy.

I do not claim that Hume would think that the world operates, on any fundamental level, like a machine. As I have established in section 2.1, Hume remains agnostic regarding any ultimate hypothetical explanations. As [author's name omitted] has argued, Hume's concept of causation tacitly assumes a mechanism. Phenomena of motion result from contact between the surfaces of bodies; there needs to be an impact, push, pull, pressure, or alike mechanical action. Bodies change their states of motions like mechanical devices generate a chain of motion. To illustrate this point, [author's name omitted] refers to the functioning of a water mill: "this [the way bodies' motions are generated] is apparent in the way that, for example, a water mill is used to crush grains. There is a succession of physical contacts among the parts of the machine."

\section{Conclusion}

This article assessed Hume's position on the intelligibility of natural philosophy in the light of the debate between Newton and Leibniz on universal gravitation. It should be concluded that Hume's philosophy encompasses a nuanced, twofold view of intelligibility. Section 2.1 indicated that he declines Leibniz's intelligibility principle. There is no ultimate reason as to why a property is ascribable of an entity. Predications are not founded on reason but on experience. Universal gravitation is no exception. In this sense, Hume has no problem in subscribing to Newtonian gravity. Long-range and instant causal action is in many ways unintelligible. But both Newton and Hume maintain that causal intelligibility should not be an ideal for the study of nature. All the same, section 2.2 provided a critical rendition of Hume's rules for causation-rules that are required to distinguish between mere regularity and causal action-and showed that the rules exhibit a mechanical intelligibility. Contiguity, succession and constant conjunction typify mechanistic interactions among bodies. These rules take their model from collisions of hard 
objects or workings of machines. Such mechanistic model is inconsistent with Newton's dynamics in which forces act between centers of mass. Although Hume rejects a core aspect of Leibniz's rationalism in denying sufficient reason, his philosophy of causation nevertheless retains a residue of mechanistic physics which Leibniz praised as being the intelligible natural philosophy. In Hume's view, the law of gravity is perfectly regular in its operation, but Newton's theory of gravity does not give a full causal account of the law as it does not include a reference to spatial contiguity and temporal priority.

\section{Acknowledgements:}

I wrote this paper when I was a visiting scholar at UCLA's Philosophy Department. Big thanks to participants of UCLA's History of Philosophy Workshop, UCSD's History of Philosophy Roundtable, LUC's History of Philosophy Roundtable, and Scientiae Conference at the University of Minnesota. Funding for my research was provided by the Finnish Cultural Foundation and Alfred Kordelin Foundation, coordinated by the Foundations' Doc Pool.

\section{References:}

Anstey, Peter (2015) "Experimental Pedagogy and the Eclipse of Robert Boyle in England." Intellectual History Review 25 (1), 115-131.

Barfoot, Michael (1990) "Hume and The Culture of Science in The Early Eighteenth Century." In Stewart M.A. (ed.) Studies in the Philosophy of Scottish Enlightenment, 151-190. Oxford: Oxford University Press.

Belkind, Ori (2012) "Newton's scientific method and the universal law of gravitation." In Janiak, Andrew, Schliesser, Eric (eds.) Interpreting Newton. Critical Essays, 138-168. Cambridge: Cambridge University Press. 
Berkeley, George (1709/1948) “An Essay Towards a New Theory of Vision.” In Luce A. A. and Jessop, T. E. (eds.) The Works of George Berkeley, the Bishop of Cloyne, Volume 1, 171-236. London: Thomas Nelson and Sons.

Biener, Zvi (2017) "De Gravitatione Reconsidered: The Changing Significance of Experimental Evidence for Newton's Metaphysics of Space.” Journal of the History of Philosophy 55 (4), 583608.

Brown, Charlotte Randall and William Edward Morris (2012) Starting with Hume. London and New York: Continuum.

Boehm, Miren (2013) "The Normativity of Experience and Causal Belief in Hume's Treatise." Hume Studies 39 (2), 203-231.

Boehm, Miren (2016) "Hume's Foundational Project in the Treatise." European Journal of Philosophy 24 (1), 55-77.

Buckle, Stephen (2004) Hume's Enlightenment Tract. Oxford: Clarendon Press.

Capaldi, Nicholas (1975) David Hume: the Newtonian Philosopher. Boston: Twayne Publishers.

Burnham, Douglas (2018) “Gottfried Leibniz: Metaphysics" Internet Encyclopedia of Philosophy, ISSN 2161-0002, http://www.iep.utm.edu/leib-met/.

Dear, Peter (2006) The Intelligibility of Nature. How Science Makes Sense of the World. Chicago: University of Chicago Press.

Della Rocca, Michael (2017) "Playing with Fire: Hume, Rationalism, and a Little Bit of Spinoza." In Della Rocca, M. (ed.) The Oxford Handbook of Spinoza, 464-481. New York: Oxford University Press. 
Demeter, Tamás (2014) "Enlarging the Bounds of Moral Philosophy: Newton's Method and Hume's Science of Man." In Biener, Zvi and Schliesser, Eric (eds.) Newton and Empiricism, 171206. New York: Oxford University Press.

Demeter, Tamás (2017) David Hume and the Culture of Scottish Newtonianism: Methodology and Ideology in Enlightenment Inquiry. Boston: Brill.

De Regt, Henk W. (2017) Understanding Scientific Understanding. New York: Oxford University Press.

De Pierris, Graciela (2006) "Hume and Locke on Scientific Methodology: The Newtonian Legacy." Hume Studies 32 (2), 277-330.

Descartes, René (1644/1982) Principles of Philosophy. Valentine Rodger Miller, Reese P. Miller (eds. trans.). Dordrecht, Boston, London: Kluwer Academic Publishers.

Dicken, Paul (2011) “On Some Limitations of Humean Disagreement: Miraculous Testimony and Contrary Religions." Sophia 50 (3), 345-355.

Downing, Lisa (2005) "Occasionalism and Strict Mechanism: Malebranche, Berkeley, Fontenelle." In Mercer, Christia, O’Neill, Eileen (eds.) Early Modern Philosophy: Mind, Matter, and Metaphysics. New York: Oxford University Press, pp. 206-230.

Ducheyne, Steffen (2009) “'Newtonian' Elements in Locke, Hume, and Reid, or: How far can One Stretch a Label?"' In Snobelen, Steven D. (ed) Enlightenment and Dissent. Isaac Newton in the Eighteenth Century, no. 25, pp 62-105.

Ducheyne, Steffen (2012) The Main Business of Natural Philosophy. Isaac Newton's NaturalPhilosophical Methodology. Dordrecht, Heidelberg, London, New York: Springer. 
Dunlop, Katherine (2012) "What geometry postulates: Newton and Barrow on the relationship of mathematics to nature." In Janiak, Andrew and Schliesser, Eric (eds.) Interpreting Newton: Critical Essays, 69-102. Cambridge: Cambridge University Press.

Force, James E. (1987) “Hume's Interest in Newton and Science.” Hume Studies 13 (2), 166-216.

Garber, Daniel and Sophie Roux (2013) “Introduction.” In Garber, Roux (eds.) The Mechanization of Natural Philosophy, xi-xviii. Dordrecht: Springer.

Harper, William (2011) Isaac Newton's Scientific Method. Turning Data into Evidence about Gravity and Cosmology. New York: Oxford University Press.

Harper, William (2016) "Newton's argument for universal gravitation.” In Rob Iliffe, George E. Smith (eds.) Cambridge Companion to Newton, Second Edition, 229-260. Clays, St Ives plc: Cambridge University Press.

Hazony, Yoram and Eric Schliesser (2016) “Newton and Hume." In Russell, Paul (ed.) The Oxford Handbook of Hume, 673-707. New York: Oxford University Press.

Henry, John (2011) "Gravity and De gravitatione: the development of Newton's ideas on action at a distance." Studies in History and Philosophy of Science 42, 11-27.

Hight, Marc A. (2008) Idea and Ontology. An Essay in Early Modern Metaphysics of Ideas. University Park, Pennsylvania: The Pennsylvania State University Press.

Hume, David (2000) A Treatise of Human Nature. Edited by David Fate Norton and Mary J. Norton. New York: Oxford University Press.

Hume, David (2006) An Enquiry concerning Human Understanding. Edited by Tom L. Beauchamp. New York: Oxford University Press. 
Hume, David (2006) An Enquiry concerning the Principles of Morals. Edited by Tom L. Beauchamp. New York: Oxford University Press.

Janiak, Andrew (2007) "Newton and the Reality of Force." Journal of the History of Philosophy 45 (1), 127-147.

Janiak, Andrew (2008) Newton as Philosopher. New York: Cambridge University Press.

Janiak, Andrew (2013) "Metaphysics and Natural Philosophy in Descartes and Newton." Foundations of Science 18, 403-417.

Kochiras, Hylarie (2011) “Gravity's cause and substance counting: contextualizing the problems.” Studies in History and Philosophy of Science 42, 167-184.

Kochiras, Hylarie (2013) "The Mechanical Philosophy and Newton's Mechanical Force." Philosophy of Science 80 (4), 557-578.

Kochiras, Hylarie (2017a) "Locke's Philosophy of Science." The Stanford Encyclopedia of Philosophy, Zalta EN (ed), URL = <https://plato.stanford.edu/entries/locke-philosophy-science/>

Kochiras, Hylarie (2017b) "Newton's General Scholium and the Mechanical Philosophy." <hal01442121v2>.

Landy, David (2018) Hume's Science of Human Nature. Scientific Realism, Reason, and Substantial Explanation. New York and London: Routledge.

Laudan, Larry (1981) “Thomas Reid and the Newtonian Turn of British Methodological Thought.” In Science and Hypotheses, 86-110. Springer: Dordrecht. 
Leibniz, Gottfried Wilhelm (1697/1989) "On the Ultimate Origination of Things" in Roger Ariew and Daniel Garber (eds. trans.) Philosophical Essays, 149-155. Hackett Publishing Company: Indianapolis \& Cambridge.

Leibniz, Gottfried Wilhelm (ca. 1710-1716/1989) "Against Barbaric Physics: Toward a Philosophy of What There Actually Is and Against the Revival of the Qualities of the Scholastics and Cimerical Intelligences" in Roger Ariew and Daniel Garber (eds. trans.) Philosophical Essays, 312-320. Hackett Publishing Company: Indianapolis \& Cambridge.

Leibniz, Gottfried Wilhelm (1715-1716/1989) "From the Letters to Clarke (1715-16)" in Roger Ariew and Daniel Garber (eds. trans.) Philosophical Essays, 321-346. Hackett Publishing Company: Indianapolis \& Cambridge.

Leibniz, Gottfried Wilhelm (1704/1996) New Essays concerning Human Understanding. Trans. eds. Peter Remnant and Jonathan Bennett. Glasgow: Cambridge University Press.

Malebranche, Nicolas (1674-1675/1977) Search after Truth. Trans. eds. Thomas M. Lennon and Paul J. Olscamp. Cambridge: Cambridge University Press.

McDonough, Jeffrey K. (2014) “Leibniz's Philosophy of Physics.” The Stanford Encyclopedia of Philosophy. Zalta, EN (ed), URL=<https://plato.stanford.edu/entries/leibniz-physics/>.

McMullin, Ernan (1978) Newton on Matter and Activity. Notre Dame, London: Notre Dame University Press.

Meli, Domenico Bertoloni (1993) Equivalence and Priority: Newton versus Leibniz. Oxford: Clarendon Press.

Millican, Peter (2003) “Hume's Sceptical Doubts concerning Induction.” In Millican, Peter (ed.) Reading Hume on Human Understanding. Essays on the First Enquiry, 107-174. New York: Oxford University Press. 
Millican, Peter (2007) Introduction to An Enquiry concerning Human Understanding by David Hume. New York: Oxford University Press, ix-lvi.

Millican, Peter (2017) "Hume's Fork, and his Theory of Relations." Philosophy and Phenomenological Research 95 (1), 3-65.

Newton, Isaac (1672/1974) "From a Letter to Oldenburg." In Newton, Isaac, Thayer, H.S. (ed.), Newton's Philosophy of Nature: Selections from his Writings. New York: Hafner Press, 6-7.

Newton, Isaac (1692-1693/2004) "Correspondence with Richard Bentley." In Janiak, Andrew (ed.) Newton. Philosophical Writings, 94-105. New York: Cambridge University Press.

Newton, Isaac (1704/1979) Opticks. Mineola: Dover Publications.

Newton, Isaac (1713/2004) “Correspondence with Roger Cotes.” In Janiak, Andrew (ed.) Newton. Philosophical Writings, 118-122. New York: Cambridge University Press.

Newton, Isaac (1687,1714,1723/1999) The Principia. Mathematical Principles of Natural Philosophy. Cohen, I. Bernard, Whitman, Anne (trans., eds.), Budenz, Julia (ass.). Berkeley: University of California Press.

Ott, Walter (2009) Causation and Laws of Nature in Early Modern Philosophy. New York: Oxford University Press.

Owen, David (2003) "Hume versus Price on Miracles and Prior Probabilities: Testimony and the Bayesian Calculation.” In Peter Millican (ed.) Reading Hume on Human Understanding, 335-348. New York: Oxford University Press.

Robison, Wade (2018) “Hume, Descartes, and Adam: Hume's Project." Proceedings of the 45 Annual Hume Society Conference. Budapest: Hungarian Academy of Sciences. 
Rosenberg, Alexander (1992) “Hume and the Philosophy of Science.” In Norton, David Fate (ed.), The Cambridge Companion to Hume, New York: Cambridge University Press, 64-89.

Rutherford, Donald (1992) “Leibniz’s Principle of Intelligibility.” History of Philosophy Quarterly 9 (1), 35-49.

Ryan, Todd (2003) "Hume's Argument for the Temporal Priority of Causes." Hume Studies 29 (1): $29-41$.

Schliesser, Eric (2007) “Hume’s Newtonianism and Anti-Newtonianism.” The Stanford Encyclopedia of Philosophy, Zalta EN (ed), URL = < https://plato.stanford.edu/entries/humenewton/>.

Schliesser, Eric (2009) “Hume’s Attack on Newton's Philosophy.” In Snobelen, Steven D. (ed) Enlightenment and Dissent. Isaac Newton in the Eighteenth Century, no. 25, 167-203.

Schliesser, Eric (2011) “Without God: gravity as a relational quality of matter in Newton's treatise." In Anstey, Peter and Jalobeanu, Dana (eds.) Vanishing Matter and the Laws of Motion: Descartes and Beyond, 80-102. New York: Routledge.

Schliesser, Eric (2013) "On reading Newton as an Epicurean: Kant, Spinozism and the changes to the Principia." Studies in History and Philosophy of Science 44, 416-428.

Shapiro, Alan E. (2004) "Newton's 'Experimental Philosophy." Early Science and Medicine 9 (3), 185-217.

Smeenk, Chris (Forthcoming) "Cosmology and Physical Astronomy in Newton's General Scholium.” Submitted for a volume regarding Newton's General Scholium. In Snobelen, Steven D., Ducheyne, S., Mandelbrote, S. (eds.). 
Smit, Houston (2010) "Apriority, Reason, and Induction in Hume." Journal of the History of Philosophy 48 (3), 313-343.

Smith, George E. (2016) “The methodology of the Principia.” In Rob Iliffe, George E. Smith (eds.) Cambridge Companion to Newton, Second Edition, 187-228. Clays, St Ives plc: Cambridge University Press.

Smith, George E. and Rob Iliffe (2016) "Introduction." In Rob Iliffe, George E. Smith (eds.) Cambridge Companion to Newton, Second Edition, 1-33. Clays, St Ives plc: Cambridge University Press.

Snelders, H. A. M. (1989) “Christiaan Huygens and Newton's Theory of Gravitation.” Notes and Records of the Royal Society of London 43 (2), 209-222.

Stanistreet, P. (2002) Hume's Scepticism and the Science of Human Nature. Aldershot: Ashgate.

Stein, Howard (1970) "On the Notion of Field in Newton, Maxwell, and Beyond." In Stuewer Roger H. (ed.) Historical Perspectives of Science, 264-287. Minneapolis: University of Minnesota Press.

Stein, Howard (1990) "'From the Phenomena of Motions to the Forces of Nature': Hypothesis or Deduction?" Proceedings of the Biennial Meeting of the Philosophy of Science Association 2, 209222.

Taylor, Jacqueline (2015) Reflecting Subjects: Passion, Sympathy, and Society in Hume's Philosophy. New York: Oxford University Press.

Westfall, Richard (1993) The Life of Isaac Newton. New York: Cambridge University Press. 